This is an electronic reprint of the original article. This reprint may differ from the original in pagination and typographic detail.

Author(s): Kivelä, Sami M.; Seppänen, Janne-Tuomas; Ovaskainen, Otso; Doligez, Blandine; Gustafsson, Lars; Mönkkönen, Mikko; Forsman, Jukka T.

Title: $\quad$ The past and the present in decision-making : the use of conspecific and heterospecific cues in nest site selection

Year: $\quad 2014$

Version:

Please cite the original version:

Kivelä, S. M., Seppänen, J.-T., Ovaskainen, O., Doligez, B., Gustafsson, L., Mönkkönen, M., \& Forsman, J. T. (2014). The past and the present in decision-making : the use of conspecific and heterospecific cues in nest site selection. Ecology, 95(12), 3428-3439. https://doi.org/10.1890/13-2103.1

All material supplied via JYX is protected by copyright and other intellectual property rights, and duplication or sale of all or part of any of the repository collections is not permitted, except that material may be duplicated by you for your research use or educational purposes in electronic or print form. You must obtain permission for any other use. Electronic or print copies may not be offered, whether for sale or otherwise to anyone who is not an authorised user. 


\title{
The past and the present in decision-making: the use of conspecific and heterospecific cues in nest site selection
}

\author{
Sami M. Kivelä, ${ }^{1,6}$ Janne-Tuomas Seppänen, ${ }^{2}$ Otso Ovaskainen, ${ }^{3}$ Blandine Doligez, ${ }^{4}$ Lars Gustafsson,${ }^{5}$ \\ Mikko MönKKÖnen, ${ }^{2}$ and JuKKa T. Forsman ${ }^{1,7}$ \\ ${ }^{1}$ Department of Biology, P.O. Box 3000, 90014 University of Oulu, Finland \\ ${ }^{2}$ Department of Biological and Environmental Science, P.O. Box 35, 40014 University of Jyväskylä, Finland \\ ${ }^{3}$ Department of Biosciences, Viikinkaari 1 ( P.O. Box 65), FI-00014 University of Helsinki, Finland \\ ${ }^{4}$ CNRS, Department of Biometry and Evolutionary Biology (LBBE UMR 5558), Université de Lyon, 69000 Lyon; Université Lyon 1, \\ 43 bd du 11 novembre 1918, 69622 Villeurbanne cedex, France \\ ${ }^{5}$ Department of Ecology and Genetics/Animal Ecology, Evolutionary Biology Centre, University of Uppsala, Norbyvägen 18D, \\ 75236 Uppsala, Sweden
}

\begin{abstract}
Nest site selection significantly affects fitness, so adaptations for assessment of the qualities of available sites are expected. The assessment may be based on personal or social information, the latter referring to the observed location and performance of both conspecific and heterospecific individuals. Contrary to large-scale breeding habitat selection, small-scale nest site selection within habitat patches is insufficiently understood. We analyzed nest site selection in the migratory Collared Flycatcher Ficedula albicollis in relation to present and past cues provided by conspecifics and by resident tits within habitat patches by using long-term data. Collared Flycatchers preferred nest boxes that were occupied by conspecifics in the previous year. This preference was strongest in breeding pairs where both individuals bred in the same forest patch in the previous year. The results also suggest preference for nest boxes close to boxes where conspecifics had a high breeding success in the previous year, and for nest boxes which are presently surrounded by a high number of breeding Great Tits Parus major. The results indicate social information use in nest site selection at a small spatial scale, where Collared Flycatchers use conspecific cues with a time lag of one year and heterospecific cues instantly.
\end{abstract}

Key words: Bayesian statistics; Collared Flycatcher; competition; conspecific attraction; Ficedula albicollis; heterospecific attraction; prospecting; social information.

\section{INTRODUCTION}

In spatio-temporally varying environments, selecting a breeding habitat or site is a decision that can entail strong fitness consequences. The quality of the breeding habitat or site often directly affects offspring survival and performance (Ens et al. 1992, Pärt 2001), and it may also affect the parent's future reproduction (Creighton et al. 2009). Hence, the decision of where to breed is likely to be one of the major determinants of lifetime reproductive success (Stokes and Boersma 1998), particularly in species with a low number of lifetime reproductive events.

Because of the importance of breeding habitat or site choices, animals are expected to assess the quality of the available habitats and sites before making their settlement decisions. Recent research indicates that

Manuscript received 12 November 2013; revised 23 April 2014; accepted 8 May 2014. Corresponding Editor: T. J. Valone.

${ }^{6}$ Present address: Department of Zoology, Stockholm University, SE-10691 Stockholm, Sweden.

E-mail: sami.kivela@zoologi.su.se

7 Present address: Section of Ecology, Department of Biology, University of Turku, F1-20014 Turku, Finland. animals commonly use social information, that is, the decisions (e.g., location cues) and performance of other individuals (e.g., Danchin et al. 2004, Seppänen et al. 2007) to make informed breeding site choices. An accumulating number of empirical studies demonstrate that not only conspecifics but also heterospecifics are frequently used as cues in breeding site choices (e.g., Muller et al. 1997, Danchin et al. 2001, 2004, Doligez et al. 2002, 2004b, Valone and Templeton 2002, Parejo et al. 2005, 2007, 2008, Fletcher 2007, Seppänen and Forsman 2007, Seppänen et al. 2007, 2011, SebastiánGonzáles et al. 2010, Forsman and Seppänen 2011, Battesti et al. 2012, Farrell et al. 2012). Theoretical studies predict that using social information may pay if the environment is too variable for ontogenetic induction of behavior to be adaptive, but sufficiently predictable in time or space for observations to hold value later or at other locations, and if the costs of social information acquisition and competition are not too high (Boulinier and Danchin 1997, Mönkkönen et al. 1999, Doligez et al. 2003, Fletcher 2006, Seppänen et al. 2007).

Social information use within and among species has implications on the spatial distribution of animals that 
contrast with the predictions of classical ecological theories. First, attraction to conspecifics (Stamps 1988, Wagner and Danchin 2003) and heterospecifics (Mönkkönen et al. 1990, Parejo et al. 2005) usually results in aggregations of individuals and may leave suitable habitats empty (Doligez et al. 2003), which violates the predictions of the ideal free distribution model (Fretwell and Lucas 1969). Secondly, attraction to potentially competing heterospecifics violates the competition tenet that predicts spatial segregation among species to avoid direct and indirect negative effects of overlapping resource use (e.g., MacArthur 1972, Martin 1996, Martin and Martin 2001). Rather than being inherently negative, the net fitness effect of interactions between competing species may vary from positive to negative, depending on the resolution of the trade-off between the benefits of information use and the costs of competition (Seppänen et al. 2007).

Studies of social information use in bird breeding site selection have mainly focused on the selection of a habitat patch at a large spatial scale. These studies indicate variation in timescales where information is used; breeding habitat selection may be guided by the location and performance of conspecifics in the previous year (e.g., Doligez. et al. 1999, 2002, 2004b, Nocera et al. 2006, Betts et al. 2008, Pärt et al. 2011, Farrell et al. 2012), while heterospecific cues may reflect the present habitat quality (Mönkkönen et al. 1990, Forsman et al. 2002, 2008, 2009, Thomson et al. 2003, Seppänen and Forsman 2007, Seppänen et al. 2011). In some cases, heterospecific locations in the previous year may guide breeding habitat selection as well (see Sebastián-Gonzáles et al. 2010). Social information use would be expected also in nest site choices at a small spatial scale, because nest site choice has strong fitness consequences (Martin 1996, Martin and Martin 2001). The use of social information in within-patch nest site selection has been demonstrated only recently, indicating that preference for certain nest site characteristics or locations is affected by the current preference and success of heterospecifics (Seppänen and Forsman 2007, Forsman and Seppänen 2011, Seppänen et al. 2011, Loukola et al. 2013), as well as by the past success of conspecifics (Pärt et al. 2011, Hoi et al. 2012) and possibly heterospecifics (Parejo et al. 2005).

The use of different cues potentially varies among individuals. Yearlings and dispersing individuals may have limited access to previous year's conspecific information (Doligez et al. 2002, 2004b, Parejo et al. 2007), so they probably rely more on currently available information than old and/or philopatric individuals. The propensity to use social information in decisionmaking should increase with decreasing time available (see Stöhr 1998 for mate choice context). Social information use is, thus, expected in migratory birds, as they generally are time limited in breeding decisions. The most valuable sources of current information may be heterospecifics, especially when there is breeding asynchrony between information sources and users, which is typically the case between resident and migratory species. Because residents are present throughout the year, they also probably have better knowledge about the environment than migrants, making ecologically close residents potentially important information sources for migrants.

In this study, we analyzed small-scale nest site choices of migratory Collared Flycatchers (Ficedula albicollis Temminck) within habitat patches by taking advantage of extensive long-term data on the Collared Flycatcher and the resident Great and Blue Tits (Parus major L. and Cyanistes caeruleus (L.), respectively). Collared Flycatchers are known to be attracted to habitat patches with high conspecific density and breeding success in the previous year (Doligez et al. 2002, 2004b), and to patches with relatively high current tit density (Forsman et al. 2008), and they also copy the perceived nest site preferences of tits (Seppänen and Forsman 2007). Moreover, Collared Flycatchers inspect the nests of conspecifics (Pärt and Doligez 2003, Doligez et al. 2004a) and heterospecifics (Forsman and Thomson 2008) during the breeding season. Evidence from the congeneric Pied Flycatcher (Ficedula hypoleuca Pallas) indicates that current tit clutch size affects flycatcher nest site selection (Forsman and Seppänen 2011, Seppänen et al. 2011, Loukola et al. 2013). Based on these findings, we hypothesize that Collared Flycatchers use information on past locations of conspecific nests and current locations of tit nests in their small-scale nest site selection. Existing data also suggest that Collared Flycatcher nest site selection may be guided by information on conspecific and tit past breeding success as well as current tit breeding performance. On the grounds of theoretical predictions (Seppänen et al. 2007), and the breeding asynchrony between flycatchers and tits, we expect flycatchers to use past conspecific cues and current heterospecific cues, including current tit performance information (e.g., clutch size) because the relatively early start of breeding in tits makes such information available.

We used long-term correlative data to analyze whether Collared Flycatchers are attracted to nest sites close to current and/or past conspecific and tit nests, or to nest sites at places where fledging success was high in the previous year, or to nest sites close to places with large (tit) clutch sizes, indicating high current success. We expect variation in timescales at which the different cues are used, but we do not have any a priori expectations on the spatial scales of assessing and using these cues. Thus, we explore the spatial extent of potential information use separately for each analyzed cue. We also investigate status dependency in decisionmaking, as we expect yearlings and dispersing older individuals to use only current heterospecific information, while philopatric individuals should use also past information. 


\section{Materials and Methods}

\section{Study system}

The Collared Flycatcher is a hole-nesting species, and it shares breeding habitat and nest site requirements particularly with resident Great and Blue Tits. Most tit pairs initiate breeding about two weeks before Collared Flycatchers arrive on the breeding grounds in spring. The three species have overlapping resource needs (nest holes and food), and there is density-dependent interspecific competition among them (Gustafsson 1987, Sasvári et al. 1987, Forsman et al. 2008).

The study area is located in the southern part of the island of Gotland in Sweden $\left(57.00^{\circ}-57.09^{\circ} \mathrm{N}, 18.29^{\circ}-\right.$ $18.35^{\circ} \mathrm{E}$; Appendix A: Fig. A1). Discrete forest patches are provided with an abundance of nest boxes, the number of boxes having increased over the years. Data were collected during 2005-2010 from 10 forest patches, including 1202 nest boxes in total. The data set contains spatially explicit breeding data for Great Tits, Blue Tits, and Collared Flycatchers for every year, excluding the absence of flycatcher data for one patch in the first year. Nest boxes were intensively monitored during the breeding season. Each nest box was visited with an interval of at most four days, and the species occupying the nest box, the stage of nest building, as well as the number of eggs was recorded upon each visit. Birds were captured and aged on the grounds of plumage characteristics and identified in case they were already ringed. The coordinates of nest boxes were measured with GPS receivers, except for a few boxes whose coordinates had to be estimated from a map (see Appendix B).

\section{Data preparation}

Only those Collared Flycatcher breeding attempts that resulted in egg laying were included. The date of nest site selection (i.e., initiation of nest-building) could not be unambiguously deduced from the data in each case, but the date of initiation of egg laying could be determined for 1920 out of the 1979 (97\%) Collared Flycatcher breeding attempts included in the data. Therefore, a parameter $\tau$ describing the time (days) from nest site selection to egg laying was defined. In other words, nest site selection was assumed to take place $\tau$ days before the first egg was laid. We set $\tau=5$ in the analysis, but the sensitivity of the results was assessed in relation to $\tau$ by repeating the analysis (see Statistical analyses) with $\tau=2$ and $\tau=8$.

Our first objective was to analyze whether Collared Flycatchers choose nest sites randomly, or are attracted to or avoid conspecifics or tits. For this purpose, the effective numbers of conspecific and tit neighbors were first calculated for each available (empty) nest box on each day during the breeding season. $O(x, \alpha, i, k, t, T-\Delta)$ denotes the effective number of neighbors (number of neighbors weighted by inverse distance) of species $x$ ( $x=$ Collared Flycatcher [CF], Great Tit [GT], Blue Tit [BT]) for nest box $i$ in the forest patch $k$ on day $t$ in year $T-$
$\Delta(T=2005, \ldots, 2010) . \Delta=0$ refers to the situation on the day of nest site choice (day $t$ in year $T$ ), and $\Delta=1$ refers to the situation at the end of the previous breeding season (year $T-1$ ). Note that $T-\Delta$ indicates which breeding season's nests are included in the neighborhood, whereas the day $t$ always refers to the date of nest site choice in year $T$ when the choice takes place. $O(x, \alpha, i, k, t, T-\Delta)$ was calculated as (see Appendix A: Fig. A2 for illustration)

$$
O(x, \alpha, i, k, t, T-\Delta)=\sum_{j=1}^{n_{k(T-\Delta)}} e^{-\frac{d_{i j}}{\alpha}} O(x, j, k, t, T-\Delta)
$$

where $d_{i j}$ is the distance between the focal nest box $i$ and a nest box $j\left(j=1, \ldots, n_{k(T-\Delta)}\right.$, where $n_{k(T-\Delta)}$ is the number of nest boxes in the forest patch $k$ in year $T-\Delta$ ) of forest patch $k$, and parameter $\alpha$ determines the spatial scale over which number ${ }_{d_{i j}}$ of neighbors is evaluated. The weighting coefficients $\left(e^{-\frac{d_{i j}}{\alpha}}\right)$ for each nest box located in the forest patch $k$ decrease exponentially with increasing distance, and the rate of decrease is affected by the parameter $\alpha$ (Appendix A: Fig. A3). Small values of $\alpha$, thus, mean that only nest boxes close to the focal box are included in the effective neighborhood, as the weights rapidly approach zero with increasing distance. As the value of $\alpha$ increases, increasingly distant nest boxes get a non-negligible weight, increasing the effective area over which number of neighbors is calculated. As we had no a priori information about the value of $\alpha$, we determined it during the statistical analysis (see Statistical analyses). The parameter $O(x, j, k, t, T-\Delta)=1$ if the nest box $j$ was occupied by species $x$ (on day $t$, if $\Delta=0$ ) in the breeding season $T-\Delta$, and otherwise $O(x, j, k, t, T-\Delta)=0$. The effective numbers of neighbors (Eq. 1) were calculated for the situation at the assumed time of nest site selection ( $\tau$ days before the first egg was laid for $\Delta=0$ ), and for the situation at the end of the previous breeding season $(\Delta=1)$.

The effective numbers of neighbors of those nest boxes that Collared Flycatchers selected were compared to the average effective numbers of neighbors of the boxes that were available for them (including the selected one) in the same forest patch on the day when they were assumed to make the choice ( $\tau$ days before the first egg was laid). This was done by calculating the difference variable

$$
\begin{aligned}
D_{O}(x, \alpha, i, k, t, T-\Delta)= & O(x, \alpha, i, k, t, T-\Delta) \\
& -E[O(x, \alpha, k, t, T-\Delta)]
\end{aligned}
$$

where $E[O(x, \alpha, k, t, T-\Delta)]$ is the expected (i.e., average) effective number of species $x(x=\mathrm{CF}, \mathrm{GT}, \mathrm{BT})$ neighbors in year $T-\Delta$ (cf. Delgado et al. 2014). The expected value was calculated by averaging the effective numbers of neighbors of all available (empty) nest boxes in the forest patch $k$ on day $t$ in year $T$.

To control for potential Collared Flycatchers' intrinsic preference for certain locations and microhabitats, 
$E[O(x, \alpha, k, t, T-\Delta)]$ was calculated as a weighted average, the nest boxes being weighted with their probabilities of being occupied by Collared Flycatchers during the years 1990-2000. These years were selected to avoid the same individuals from occurring in both data sets, and because older data may not reliably reflect habitat quality in the study period due to succession and forestry. Nest boxes for which data were missing for 1990-2000 were assumed to have been occupied with average probability over those nest boxes with data for forest patch $k$.

Our second objective was to analyze the influence of conspecific and heterospecific breeding performance on Collared Flycatcher nest site selection. Thus, the effective average number of fledglings $F(x, \alpha, i, k, t, T-$ 1) in the neighborhood of the focal nest box produced by species $x(x=\mathrm{CF}, \mathrm{GT}$; there is no proper data on Blue Tits) in the previous breeding season (year $T-1$ ) was calculated, as well as the effective average clutch size $C(x, \alpha, i, k, t, T)$ of species $x(x=\mathrm{GT}, \mathrm{BT})$ on day $t$ in the current breeding season (year $T$ ). These were calculated as

$$
F(x, \alpha, i, k, t, T-1)=\frac{\sum_{j=1}^{n_{k(T-1)}} e^{-\frac{d_{i j}}{\alpha}} f(x, j, k, t, T-1)}{\sum_{j=1}^{n_{k(T-1)}} e^{-\frac{d_{i j}}{\alpha}} O(x, j, k, t, T-1)}
$$

and

$$
C(x, \alpha, i, k, t, T)=\frac{\sum_{j=1}^{n_{k T}} e^{-\frac{d_{i j}}{\alpha}} c(x, j, k, t, T)}{\sum_{j=1}^{n_{k T}} e^{-\frac{d_{i j}}{\alpha}} O(x, j, k, t, T)} .
$$

Here, $f(x, j, k, t, T-1)$ and $c(x, j, k, t, T)$ are the numbers of fledglings and eggs produced by species $x$ in the nest box $j$ of the forest patch $k$. Note that the performance measures given by Eqs. 3 and 4 are averages (average numbers of fledglings or eggs per nest in the effective neighborhood) contrary to the number of neighbors (Eq. 1) and, therefore, the weighted sums of fledglings and eggs in the neighborhoods are divided by the effective numbers of neighbors. As for the number of neighbors, difference variables were calculated as

$$
\begin{aligned}
D_{F}(x, \alpha, i, k, t, T-1)= & F(x, \alpha, i, k, t, T-1) \\
& -E[F(x, \alpha, k, t, T-1)]
\end{aligned}
$$

and

$$
D_{C}(x, \alpha, i, k, t, T)=C(x, \alpha, i, k, t, T)-E[C(x, \alpha, k, t, T)]
$$

where $E[F(x, \alpha, k, t, T-1)]$ and $E[C(x, \alpha, k, t, T)]$ are the expected numbers of fledglings in the previous year and eggs in the current season produced by species $x$ in the neighborhoods of the available nest boxes in the forest patch $k$ on day $t$ in year $T$. The calculation of $E[F(x, \alpha, k, t, T-1)]$ and $E[C(x, \alpha, k, t, T)]$ was weighted with Collared Flycatcher occupancy probabilities in the same way as for $E[O(x, \alpha, k, t, T-\Delta)]$. Tit clutch size information was included in $C(x, \alpha, i, k, t, T)$ only when tit eggs were recorded to be uncovered, because tits often cover their eggs with nest material during the laying period, but not during incubation. Thus clutch size information becomes available to Collared Flycatchers at the start of incubation.

As individual-level variation in access to different cues and their use is expected (Doligez et al. 2002, 2004b, Parejo et al. 2007), the information on Collared Flycatcher age (yearling/old) and dispersal status (immigrant/philopatric) was combined as a single "status" variable that contained three classes: young, immigrant, and philopatric. These classes well capture individual access to different cues. All yearlings were assigned the status "young." Old individuals were assigned the status "philopatric" in year $T$ if they were observed breeding in the same forest patch in years $T$ and $T-1$, and "immigrant" otherwise. A status was defined only for those individuals that could be unambiguously assigned to one of the three classes.

To take repeated measures on the same individuals into account and to test for the influence of status on nest site selection, analyses were restricted to observations for which both female and male identities (ring numbers) and statuses (young, immigrant, or philopatric) were known. There were 1144 such observations, but the response variables contained missing values, reducing the variable-specific data available for analysis (see Table 1). For treatment of missing data and special cases, see Appendix B.

\section{Statistical analyses}

The differences between realized and expected neighbor number $\left(D_{O}(x, \alpha, i, k, t, T-\Delta)\right)$ and breeding performance $\left(D_{F}(x, \alpha, i, k, t, T-1)\right.$ and $\left.D_{C}(x, \alpha, i, k, t, T)\right)$ variables were treated as response variables in the analyses (10 variables in total; Table 1). As the response variables were constructed as the difference between the realized number of neighbors (or performance in the neighborhood) and its expectation (Eqs. 2, 5, and 6), values greater or smaller than 0 indicate selection of nest boxes with larger or smaller numbers of neighbors (or neighborhoods with higher or lower performances) than expected at random (cf. Delgado et al. 2014), respectively. Hence, we analyzed whether the data on each response variable were consistent or inconsistent with the null hypothesis of random nest site choices. In cases of nonrandom nest site choices, the directions of deviations from the null hypothesis were also of interest to us, as well as the spatial scales where different cues were used.

To facilitate comparisons among the 10 analyzed response variables, these variables, denoted by $Y_{i}$ below, 
TABLE 1. The optimal values of the spatial scale parameter $\alpha$ (Eqs. 1, 3, and 4), the proportions of total variance explained $\left(R^{2}\right)$, the spatial accuracy of the parameter $\alpha$, and the mean value of the variable and its (frequentist $\dagger$ ) confidence interval for the 10 analyzed variables explaining Collared Flycatcher $(\mathrm{CF})$ nest site choice.

\begin{tabular}{|c|c|c|c|c|c|c|c|c|}
\hline \multirow[b]{2}{*}{ Normalized difference in } & \multirow[b]{2}{*}{$N$} & \multirow[b]{2}{*}{$\alpha(\mathrm{m})$} & \multirow[b]{2}{*}{$R^{2}$} & \multicolumn{2}{|c|}{$\begin{array}{c}\text { Interval of } \alpha \text { where } R^{2} \\
\text { is close to maximum }(\mathrm{m}) \dagger\end{array}$} & \multirow[b]{2}{*}{ Mean } & \multicolumn{2}{|c|}{$99.5 \%$ confidence interval } \\
\hline & & & & Min & Max & & Lower bound & Upper bound \\
\hline $\begin{array}{l}\text { CF neighbors } T \\
D_{O}(\mathrm{CF}, 7, i, k, t, T)\end{array}$ & 1101 & 7 & 0.129 & 5 & 17 & -0.222 & -0.305 & -0.140 \\
\hline $\begin{array}{l}\text { CF neighbors } T-1 \\
D_{O}(\mathrm{CF}, 1, i, k, t, T-1)\end{array}$ & 1093 & 1 & 0.0925 & 1 & 19 & 0.263 & 0.181 & 0.345 \\
\hline $\begin{array}{l}\mathrm{CF} \text { success } T-1 \\
\quad D_{F}(\mathrm{CF}, 83, i, k, t, T-1)\end{array}$ & 1058 & 83 & 0.0636 & 1 & 101 & 0.205 & 0.120 & 0.289 \\
\hline $\begin{array}{l}\text { GT neighbors } T \\
\quad D_{O}(\mathrm{GT}, 11, i, k, t, T)\end{array}$ & 1097 & 11 & 0.0855 & 1 & 21 & 0.227 & 0.145 & 0.310 \\
\hline $\begin{array}{l}\text { GT neighbors } T-1 \\
\quad D_{O}(\mathrm{GT}, 75, i, k, t, T-1)\end{array}$ & 1109 & 75 & 0.107 & 23 & 101 & -0.290 & -0.371 & -0.209 \\
\hline $\begin{array}{l}\text { GT success } T-1 \\
\quad D_{F}(\mathrm{GT}, 101, i, k, t, T-1)\end{array}$ & 1109 & 101 & 0.0320 & 1 & 101 & 0.0779 & -0.0063 & 0.162 \\
\hline $\begin{array}{l}\text { GT clutch } T \\
\qquad D_{C}(\mathrm{GT}, 1, i, k, t, T)\end{array}$ & 1096 & 1 & 0.0448 & 1 & 101 & -0.0473 & -0.132 & 0.0376 \\
\hline $\begin{array}{l}\text { BT neighbors } T \\
D_{O}(\mathrm{BT}, 1, i, k, t, T)\end{array}$ & 1063 & 1 & 0.126 & 1 & 1 & -0.244 & -0.328 & -0.161 \\
\hline $\begin{array}{l}\text { BT neighbors } T-1 \\
\quad D_{O}(\mathrm{BT}, 101, i, k, t, T-1)\end{array}$ & 882 & 101 & 0.0522 & 59 & 101 & -0.155 & -0.249 & -0.0618 \\
\hline $\begin{array}{l}\text { BT clutch } T \\
\quad D_{C}(\mathrm{BT}, 101, i, k, t, T)\end{array}$ & 1063 & 101 & 0.0434 & 1 & 101 & 0.00683 & -0.0795 & 0.0931 \\
\hline
\end{tabular}

Notes: Great and Blue Tits are denoted by GT and BT, respectively, number of neighbors by "neighbors," breeding success by "success," clutch size by "clutch," current year by $T$ and the previous year by $T-1 . N$ is number of observations.

$\dagger$ The confidence interval of the mean was calculated for the raw data; it is not based on the Bayesian analysis. The confidence intervals were adjusted for multiple (10 variables) testing by assuming a risk level of 0.05 (i.e., $95 \%$ confidence intervals), and calculating $100 \times(1-0.05 / 10) \%=99.5 \%$ confidence intervals instead of the $95 \%$ ones.

$\ddagger$ The endpoints of the range of $\alpha$ where $R^{2} \geq 3 / 4 \max \left(R^{2}\right)$.

were normalized into the variables $y_{i}$ so that, for the latter ones, the average square sum was unity, i.e.,

$$
y_{i}=\sqrt{\frac{n}{\sum_{i=1}^{n} Y_{i}^{2}}} Y_{i},
$$

where the index $i$ runs over all the $n$ observed Collared Flycatcher nest site choices.

All analyses were performed within the Bayesian framework. First, the spatial scale (the value of the parameter $\alpha$ ) of the analysis was determined separately for each response variable. To do so, the data were calculated with values of $\alpha$ ranging from $1 \mathrm{~m}$ (only the selected box is included in the neighborhood) to $101 \mathrm{~m}$ (practically all boxes in a forest patch are included in the neighborhood) with an increment of $2 \mathrm{~m}$. Then, an identical statistical model (Appendix B) was fitted to each data set with the R function "MCMCglmm" (Hadfield 2010) that utilizes Markov chain Monte Carlo (MCMC) methods in R version 2.13.1 (R Development Core Team 2011). Each of these univariate models contained the date of nest site choice (April day, continuous variable; 1 April is day 1) and the statuses (young, immigrant, or philopatric) of the female and the male of the breeding pair and all possible interactions among them as fixed effects. The date of nest site choice was included as a covariate because it negatively correlates with time available for nest site selection, and is thus expected to affect the use of social cues (cf. Stöhr 1998). Female and male identities (ring numbers) as well as box identity were set as random factors to control for repeated measures. To take into account possible individual-level variation in behavior in relation to status, status-specific random effects were defined for both female and male identities. Consequently, individual-level variance was split among the three statuses in both females and males, and all covariances were ignored (Appendix B). Inverse Wishart priors were defined for the variance components in all analyses, and the convergence of the MCMC chains was assessed by visual evaluation, and by calculating autocorrelations for the stored parameter estimates (see Appendix B for details). The MCMC chains were run for 150000 iterations. The burn-in period consisted of 30000 iterations, and the remaining 120000 iterations were thinned with an interval of 120 , resulting in 1000 samples from the posterior distribution.

The above procedure produced 51 models for each of the 10 analyzed variables, each model being associated with a different value of $\alpha$. The performances of those models were investigated in terms of the explanatory power $R^{2}$, defined as the proportion of total variance explained by the model (averaged over the posterior distribution). Thus, we define $R^{2}=\mathrm{ESS} / \mathrm{TSS}$, where TSS $=\sum_{i=1}^{n} y_{i}^{2}$ is the total sum of squares, and $\mathrm{ESS}=\sum_{i=1}^{n} \hat{y}_{i}^{2}$ is the explained sum of squares, with $\hat{y}_{i}$ denoting the fitted values. The reason why we measure $R^{2}$ by the 
squared sum instead of the variance is that the intercept is an important part of the model and inference, as it measures whether the individuals selected, on average, low or high numbers of neighbors, or neighborhoods with low or high performance (see also Delgado et al. 2014). For each of the 10 analyzed variables, the value of $\alpha$ that resulted in the best model fit in the sense of highest $R^{2}$ was selected (cf. Delgado et al. 2014). From these models fitted to the "best" data, posterior distributions for regression lines in relation to the day of nest site choice fitted to each of the nine combinations of female and male statuses were derived. Then, the $95 \%$ highest posterior density credibility intervals [function "HPDinterval" (Plummer et al. 2006)] of the regression lines were derived and inferences were based on them by comparing the credibility intervals to the expectation under the null hypothesis (i.e., 0).

\section{RESULTS}

The variable-specific spatial scales tended to be close to the extreme ends of the analyzed range of the parameter $\alpha$ (Table 1), suggesting that different cues potentially guiding nest site choices are used at either a very small or large spatial scale. The overall mean values of 7 out of the 10 analyzed variables (i.e., the difference between the selected and the expected numbers of neighbors, or neighborhood performance) were different from zero (Table 1), indicating nonrandom nest site choices in relation to these variables. The strongest positive effects (indicating attraction) were observed for the previous year's number of conspecific neighbors and conspecific breeding success as well as the present year's number of Great Tit neighbors. The strongest negative effects (indicating avoidance) were observed for the numbers of previous year's Great Tit neighbors, the present year's Blue Tit and conspecific neighbors, as well as the previous year's Blue Tit neighbors.

The fitted regression lines and their 95\% credibility intervals (Figs. 1, 2, and Appendix A: Figs. A4-A13; see Appendix A: Table A1 for parameter estimates of the regression models and their credibility intervals) indicated nonrandom nest site choices that were biologically meaningful (effect size $>0.1$ ) in all of the seven abovementioned variables (Appendix A: Figs. A4-A13). However, the strong effect size of present year's number of Blue Tit neighbors is likely to be an artifactual consequence of outliers in the data (Appendix A: Fig. A11). The three remaining variables (previous year's Great Tit breeding success in the neighborhood, and Great and Blue Tit clutch sizes in the neighborhood) that had small overall effect sizes seemed biologically unimportant. The few female and male status combinations where the regression lines indicated nonrandom nest site choices in these three variables (Fig. 1; Appendix A: Figs. A9, A10, and A13) were generated by a few deviant observations very early or late in the spring, questioning the generality of these results. Thus, we concentrate on the six variables deemed biologically meaningful in the following sections.

\section{Conspecific cues}

For nest site choices in relation to the number of neighboring previous year's conspecific nests, the optimal spatial scale $(\alpha=1 \mathrm{~m})$ was so small that essentially only the chosen nest box itself was included in the neighborhood. In particular, breeding pairs including at least one philopatric individual selected nest boxes occupied by a conspecific pair in the previous year more often than at random (Figs. 1 and 2; Appendix A: Fig. A5). This result is not a trivial consequence of individuals returning to their previous year's nest site, as only $18.8 \%$ and $25.3 \%$ of those philopatric females and males, respectively, that chose a nest box occupied by conspecifics in the previous year did so. Attraction to previous year's conspecific nests was the strongest in breeding pairs where both the female and the male were philopatric, and the attraction remained until very late in the spring (Figs. 1 and 2; Appendix A: Fig. A5). Attraction to previous year's conspecific nests was quite clear also in those breeding pairs where both individuals were older than yearlings (Fig. 1; Appendix A: Fig. A5). Weak attraction to previous year's conspecific nests was also observed in the middle of the season among immigrant males breeding with young females, but not in other status combinations (Fig. 1).

The data on nest site choices in relation to the number of previous year's conspecific neighbors becomes practically binary when $\alpha=1 \mathrm{~m}$ (i.e., neighborhood includes only the selected box), with selected nest boxes either having been occupied or not been occupied by conspecifics in the previous year (Fig. 2). Such bimodality may be problematic in a linear regression analysis, but we emphasize that the result is not an artifact. This is because the $95 \%$ credibility intervals of the fitted regression line for pairs where both individuals are philopatric indicate nonrandom attraction to boxes with a high number of conspecifics in the neighborhood in the previous year in early season up to the $\alpha$ value of $69 \mathrm{~m}$, the data being strictly unimodal with this large value of $\alpha$ (data not shown).

At a large spatial scale $(\alpha=83 \mathrm{~m})$, a weak attraction was observed to nest boxes with high conspecific breeding success in the previous year in the neighborhood. This was particularly observed in the middle of the season and for all female and male status combinations, except for breeding pairs with a young male and for pairs where both were immigrants (Fig. 1: Appendix A: Fig. A6). These results are not consequences of attraction to previous year's conspecific nests, because these two response variables are not correlated (Appendix A: Table A2).

A weak avoidance was observed at a small spatial scale $(\alpha=7 \mathrm{~m})$ in relation to current conspecific neighbors (Fig. 1 and Appendix A: Fig. A4), most likely indicating small-scale territoriality. 

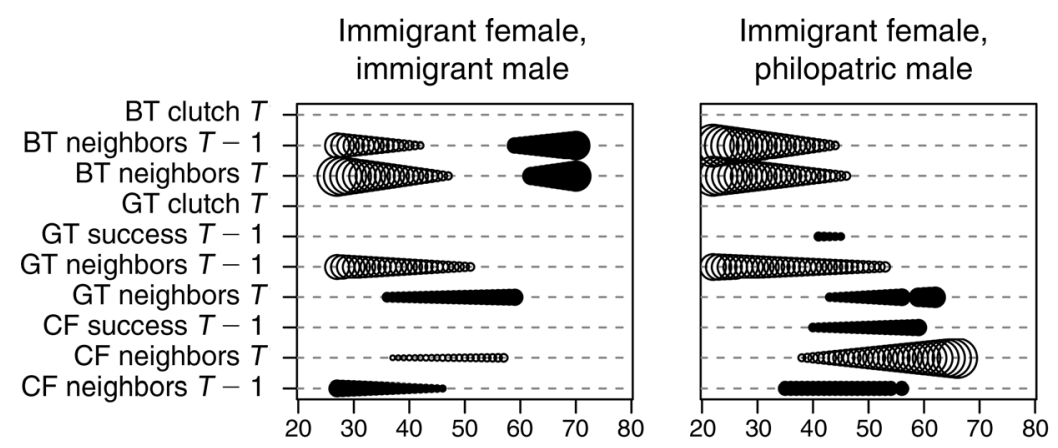

\section{Immigrant female, young male}

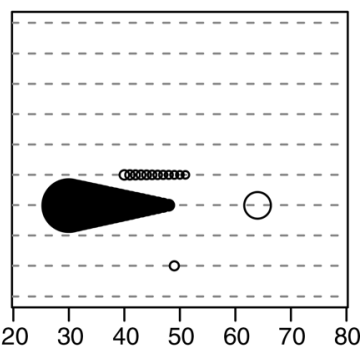

Philopatric female, immigrant male

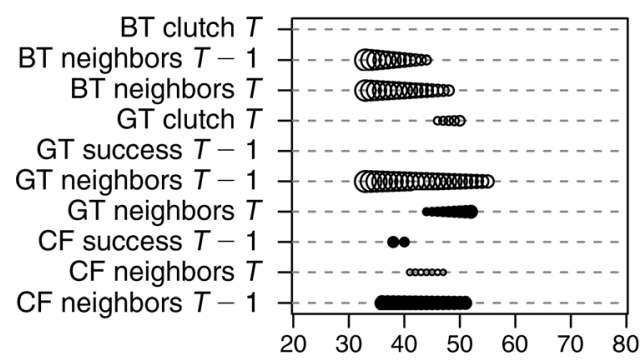

\section{Philopatric female,} philopatric male

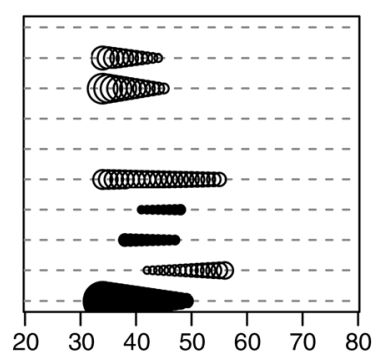

Philopatric female, young male

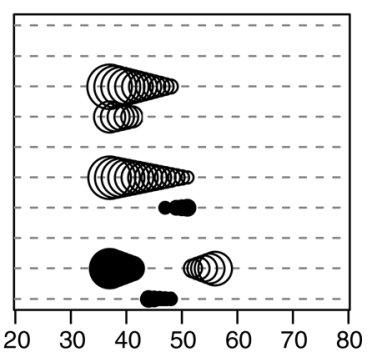

Young female, immigrant male

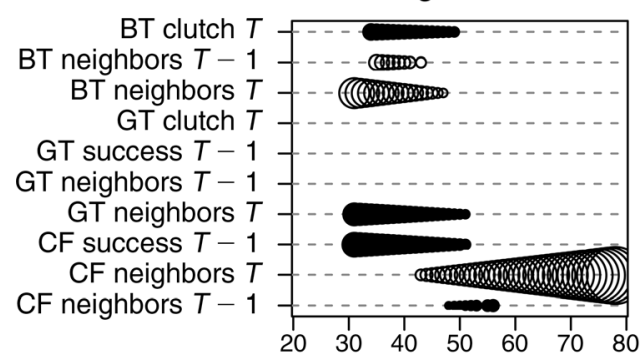

Young female, philopatric male

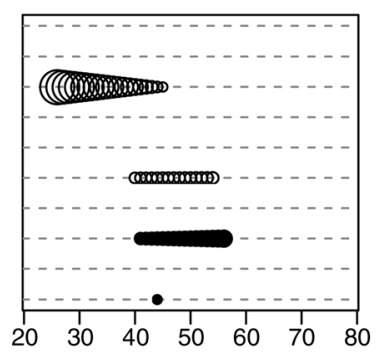

Young female, young male

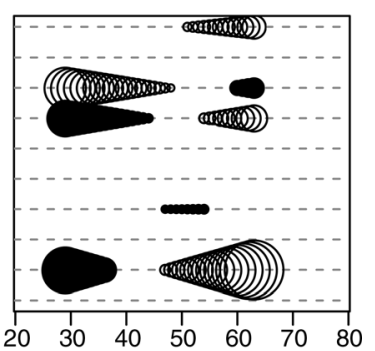

Date of nest site choice (day 1 is 1 April)

FIG. 1. A summary of nonrandom nest site choices in Collared Flycatchers. Open symbols denote negative values (i.e., avoidance) and closed symbols positive values (i.e., attraction). Increasing relative symbol size indicates increasing deviation (absolute value) of the fitted regression lines from zero. Day 1 is 1 April. Cases where the $95 \%$ credibility intervals of the fitted values include zero are not illustrated. The gray dashed lines depict the variable-specific rows in each panel. The results are shown for the 10 variables listed in Table 1 and for the nine female and male status combinations. Collared Flycatchers, Great, and Blue Tits are denoted by CF, GT, and BT, respectively, number of neighbors by "neighbors," breeding success by "success," clutch size by "clutch," current year by " $T$ " and the previous year by " $T-1$. ."

\section{Heterospecific cues}

At a spatial scale approximately including the closest neighboring nest boxes $(\alpha=11 \mathrm{~m})$, there was attraction to nest boxes with a high number of current Great Tit neighbors. This was the case especially in early and/or middle season for all status combinations, except when young females were breeding with philopatric males (Fig. 1; Appendix A: Fig. A7).

Avoidance of boxes with relatively many Great and Blue Tit neighbors in the previous year was also observed, but at a large spatial scale ( $\alpha$ was 75 and
$101 \mathrm{~m}$, respectively). Avoidance occurred in early and/or middle season in most of the female and male status combinations, particularly when both individuals of the breeding pair were old (Fig. 1; Appendix A: Figs. A8 and A12). The variables measuring nest site selection in relation to the numbers of previous year's Great and Blue Tit neighbors, indicating avoidance, are negatively correlated with the variables that indicate conspecific attraction (neighbors and breeding success in the previous year) (Appendix A: Table A2), suggesting a correlated response to the observed attraction rather than avoidance per se. 

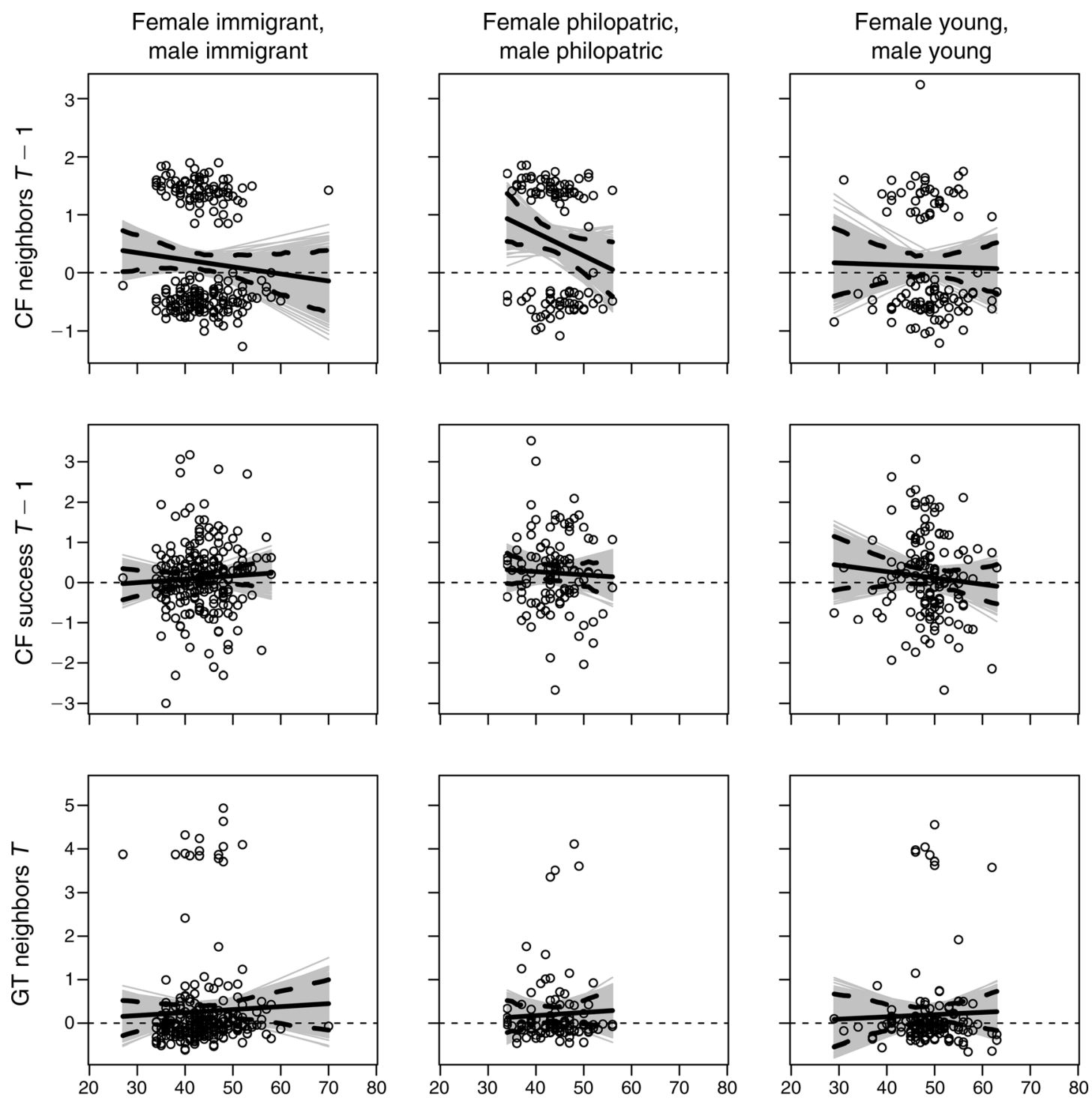

Date of nest site choice (day 1 is 1 April)

FIG. 2. The normalized difference between selected nest boxes and available nest boxes in the numbers of previous year's conspecific Collared Flycatcher neighbors (CF neighbors $T-1$; top row), in the previous year's conspecific breeding success in the neighborhood (CF success $T-1$; middle row), and in the number of Great Tit neighbors (GT neighbors $T$; bottom row) shown in relation to the date of nest site choice for those breeding pairs where both the female and the male were immigrants (left column), philopatric (the middle column), or young (right column). Data points indicate observations; Day 1 is 1 April. Thick solid lines are the fitted regression lines, thick dashed lines delimit the $95 \%$ credibility intervals of the regression lines, and gray lines indicate the posterior distributions of the regression lines. The horizontal dashed line indicates a difference of zero, which means random nest site choice. Positive and negative values indicate attraction and avoidance, respectively.

\section{(In) sensitivity of the results}

The conclusions were not sensitive to the prior distribution as indicated by the very high correlations between fixed effects (posterior means of fixed-effect parameters) of models differing with respect to the prior distribution (all correlation coefficients $>0.92$; Appendix A: Table A3; see Appendix B for prior definition). The value of the parameter $\tau(2,5$, or 8 days) had a negligible effect on 8 out of the 10 variables analyzed, as the fixed effects were very strongly correlated when estimated with the alternative values of $\tau$ (all correlation coefficients in these eight variables $>0.93$; Appendix A: Table A4). The two response variables for which the value of $\tau$ significantly affected the estimated fixed effects were Great and Blue Tit clutch sizes in the neighborhood, as indicated by weak or absent correlations between fixed effects of equal models fitted to the data that were calculated with the alternative values of $\tau$ 
(Appendix A: Table A4). Despite this, the conclusion that Great and Blue Tit clutch sizes in the neighborhood do not affect Collared Flycatcher nest site selection was insensitive to the value of $\tau$ (the $95 \%$ credibility intervals of the fitted regression lines invariably encompassed 0 ).

There was only weak evidence of spatial autocorrelation of the observations in some response variables (Appendix A: Fig. A14), indicating that the results are not confounded by spatial autocorrelations. Because the comparisons among female and male statuses as well as among different nest site selection dates were not adjusted for multiple comparisons, these results should, however, be interpreted with caution.

\section{Discussion}

Our analyses suggest that both conspecific and heterospecific cues affect small-scale (within-patch) nest site selection in the Collared Flycatcher. These cues include previous year's conspecific nest locations and breeding success, and the number of Great Tit neighbors in the current year. Philopatric Collared Flycatchers (i.e., individuals that bred in the same habitat patch in the previous year) clearly preferred those nest boxes where conspecifics were breeding in the previous year. Conspecific breeding success in the previous year affected nest site choice at a larger spatial scale (close to the habitat patch scale), so that nest boxes in areas with relatively high conspecific breeding success in the previous year were preferred. This is consistent with earlier findings on habitat patch selection in this species (Doligez et al. 1999, 2002, 2004a, b). Moreover, Collared Flycatchers seemed to be attracted to already settled Great Tits but avoided currently breeding conspecifics at a comparable spatial scale. This suggests that for Collared Flycatchers, the benefits of settling close to Great Tits (Forsman et al. 2008; see also Forsman et al. 2002) outweigh the costs of interspecific competition as long as tit density is not too high (Gustafsson 1987, Sasvári et al. 1987, Forsman et al. 2007, 2008), but intraspecific competition is always avoided. In a nutshell, our results highlight the complexity of the information-gathering and decision-making process of Collared Flycatchers; they seem to use past and present conspecific and heterospecific cues at different spatial scales.

There was strong attraction to nest boxes occupied by conspecifics in the previous year by Collared Flycatcher pairs where both the male and the female were philopatric (Fig. 2). A similar, though weaker, effect was invariably observed in breeding pairs where only one individual was philopatric. These results make sense, as philopatric individuals should have the best knowledge of conspecific nest locations in the patch where they breed. The results likely indicate social information use rather than breeding site fidelity, as only $2 \%$ and $9 \%$ of all nesting observations were such that the female or the male, respectively, returned to the same box where it bred in the previous year (see also Pärt and
Gustafsson 1989). Individuals could also be faithful to the immediate neighborhood of the previous nest site. However, the spatial scale practically including only the selected nest box is expected if there is social information use at the nest site scale, but not if there is only fidelity to the neighborhood of the previous nest site.

Determining nest locations (location cues) may be easy compared to assessing the success of individual nests (performance cues). Thus, birds may need to use the number of fledglings in the neighborhood or in a habitat patch as performance information instead of nest-specific performance information. This may explain the larger spatial scale at which attraction to neighborhoods with high conspecific breeding success in the previous year took place compared to that in the previous year's conspecific neighborhood. The large spatial scale in using conspecific performance cues is also in line with earlier studies (Doligez et al. 2002, 2004b; see also Parejo et al. 2007 for a similar trend in the Blue Tit).

At a small spatial scale, there was territorial exclusion and/or avoidance of conspecifics, as well as attraction to a competing species, the Great Tit. It is likely that this result does not stem from similar microhabitat preferences in flycatchers and Great Tits, because experimental studies have demonstrated that migratory passerines, including the Collared Flycatcher, are attracted to manipulated presence and high density of tits (Mönkkönen et al. 1990, Forsman et al. 2002, 2008, 2009, Thomson et al. 2003), and both Collared and Pied Flycatchers copy the nest site choices of tits (Seppänen and Forsman 2007, Forsman and Seppänen 2011, Loukola et al. 2013), strongly suggesting that heterospecific attraction underlies the observed results.

Despite attraction to Great Tits, Great Tit clutch size did not affect Collared Flycatchers' nest site selection, although flycatchers inspect tit nests at the time of laying and incubation (Merilä and Wiggins 1995; J. T. Forsman, personal observation), and tit clutch size has been shown to affect the choice of nest site characteristics in the Pied Flycatcher (Forsman and Seppänen 2011, Seppänen et al. 2011, Loukola et al. 2013). Either Collared Flycatchers do not use tit clutch size information in their nest site choices, or other cues guiding nest site choices outweigh tit clutch size information when such cues are available. The mortal risk of inspecting tit nests (Merilä and Wiggins 1995) may also decrease the profitability of clutch size information.

Collared Flycatchers appeared to prefer nest boxes surrounded by smaller than average numbers of Great and Blue Tit nests in the previous year, which seems contradictory given the observed attraction to currently breeding Great Tits. However, the correlations among the analyzed variables (Appendix A: Table A2) suggest that Collared Flycatchers do not use past locations of tit nests as cues in their nest site choices, but the result only represents a correlated response to the observed patterns of attraction. 
Integration and use of different cues for nest site selection

The results suggest individual-level variation in the access to different sources of social information, and consequently, in social information use for nest site selection at a small spatial scale. Philopatric individuals with the best knowledge about their breeding habitat patch, including the locations of conspecific and heterospecific nests from the previous year, were strongly attracted to previous year's conspecific nests. In contrast, fledglings probably have more limited time and experience in prospecting cues for choosing a nest site in the next year than adults (Doligez et al. 2004b, Parejo et al. 2007). Moreover, yearling Collared Flycatchers arrive to the breeding grounds later than older individuals, as suggested by their later nest site choices (mean dates [April day] of nest site choice [ $\tau=$ 5] in females; young, 47.5 [95\% CI, 46.9, 48.1]; old, 44.9 [44.5, 45.3]; and males; young, 48.2 [47.3, 49.0]; old, $44.5[44.0,45.0])$. Later arrival of yearlings may further limit their possibilities to settle on preferred sites, because tits and earlier-arriving old flycatchers already have occupied part of the nest sites. Yearlings and old dispersing individuals may not have access to conspecific cues (presence and success) available in the previous year in the patch they select (see also Doligez et al. 2002, 2004b), so they may rely on currently available heterospecific cues. Yet our results suggest that the use of current heterospecific location cues is not restricted to yearlings and dispersing old individuals, but also philopatric individuals may use those cues, probably as a secondary option if the nest sites preferred on the grounds of past conspecific cues have already been occupied. However, even if previous year's conspecific cues cannot be used for nest site selection by all individuals, the current presence and/or density of conspecifics may be used as cues for largescale patch selection. Such conspecific attraction at the habitat patch scale has been found in the Collared Flycatcher (Doligez et al. 2002, 2004b) and other birds (Muller et al. 1997, Fletcher 2007, Farrell et al. 2012). Thus, it may be a general phenomenon.

Attraction to heterospecifics sharing the same resources is evolutionarily stable if the benefits of settling close to heterospecific competitors, on average, exceed the costs of interspecific competition (Seppänen et al. 2007). For migratory birds, the time saving resulting from using residents as cues may have a large fitness benefit, as delayed breeding is costly (Wiggins et al. 1994, Siikamäki 1998). This may lead to strongly asymmetric consequences of co-occurrence, which appears to be the case between the Pied Flycatcher and the Great Tit: tits suffer from proximity to flycatchers even at low densities (Forsman et al. 2007), while the interaction turns negative for flycatchers only at high tit densities (Gustafsson 1987). Intraspecific competition is expected to be stronger and more symmetric than interspecific competition, so settling close to conspecifics is probably more costly than settling close to heterospecifics. Attraction to heterospecifics has also been observed in breeding and foraging patch selection in many other vertebrate and invertebrate systems (e.g., Hodge and Storfer-Isser 1997, Coolen et al. 2003, Parejo et al. 2005, 2008, Fletcher 2007, Sebastián-Gonzáles et al. 2010), implying that location of heterospecifics commonly impact the spatial distribution of animals resulting in multispecies aggregations.

The results of this and other studies suggest integrated use of different cues in nest site selection at a small spatial scale. Time limitation and a high degree of synchrony in migratory birds' nest site choices preclude the utilization of current conspecific performance information in nest site choices (Doligez et al. 2004a, Nocera et al. 2006). Therefore, information on conspecific performance and on the location of successful nests may generally be collected in the previous year (see Nocera et al. 2006, Betts et al. 2008, Pärt et al. 2011, Farrell et al. 2012, Hoi et al. 2012). Such information can be successfully used in the next year, if there is sufficient temporal autocorrelation in breeding conditions within habitat patches (Boulinier and Danchin 1997, Doligez et al. 1999, 2003). If temporal autocorrelation in breeding conditions is low, current heterospecific information may be more valuable than degraded past conspecific information.

\section{Conclusions}

Our results indicate that social information is used by a migratory bird in nest site choices at a small spatial scale within habitat patches. The results indicate conspecific attraction (Stamps 1988), but there is a time lag of one year between information acquisition and utilization. A source of instant social information seems to be a resident competing species, resulting in heterospecific attraction (Mönkkönen et al. 1990, Parejo et al. $2005)$ at the nest site scale. These results fit well with the theoretical prediction that the net fitness benefit of social information use peaks at some temporal or ecological distance from the source of the information (Seppänen et al. 2007). The spatial accuracy of information depends strongly on whether the information comes from presence (location cues) or performance of other individuals; it is high for past and current nest locations, but low for performance information (breeding success) in the studied non-colonial and territorial species. Finally, our study emphasizes that both the present and the past information, as well as avoidance and attraction to conspecifics and heterospecific competitors, affect the spatial distribution of animals, which has implications for the development of ecological theory and basic and applied empirical research.

\section{Acknowledgments}

We thank Peer 522 in Peerage of Science and two anonymous reviewers for helpful comments on an earlier version of the manuscript. We also thank the numerous people that have been involved in collecting the data and the landowners of Gotland 
for allowing us to work on their property. This study was financed by the Academy of Finland (grants No. 122665 and No. 125720 to J. T. Forsman and No. 250444 to O. Ovaskainen), the European Research Council (ERC StG 205905 to O. Ovaskainen), the French National Research Agency (ANR-06-JCJC0082 to B. Doligez), the CNRS (PICS No. 3054 and 31520 and PEPS to B. Doligez) and by numerous grants from the Swedish Research Council and FORMAS (to L. Gustafsson).

\section{Literature Cited}

Battesti, M., C. Moreno, D. Joly, and F. Mery. 2012. Spread of social information and dynamics of social transmission within Drosophila groups. Current Biology 22:309-313.

Betts, M. G., A. S. Hadley, N. Rodenhouse, and J. J. Nocera. 2008. Social information trumps vegetation structure in breeding-site selection by a migrant songbird. Proceedings of the Royal Society B 275:2257-2263.

Boulinier, T., and E. Danchin. 1997. The use of conspecific reproductive success for breeding patch selection in terrestrial migratory species. Evolutionary Ecology 11:505-517.

Coolen, I., Y. V. Bergen, R. L. Day, and K. N. Laland. 2003. Species difference in adaptive use of public information in sticklebacks. Proceedings of the Royal Society B 270:24132419.

Creighton, J. C., N. D. Heflin, and M. C. Belk. 2009. Cost of reproduction, resource quality, and terminal investment in a burying beetle. American Naturalist 174:673-684.

Danchin, E., L. A. Giraldeau, T. J. Valone, and R. H. Wagner. 2004. Public information: from noisy neighbours to cultural evolution. Science 305:487-491.

Danchin, E., D. Heg, and B. Doligez. 2001. Public information and breeding habitat selection. Pages 243-258 in J. Clobert, E. Danchin, A. A. Dhondt, and J. D. Nichols, editors. Dispersal. Oxford University Press, New York, New York, USA.

Delgado, M. D., V. Penteriani, J. M. Morales, E. Gurarie, and O. Ovaskainen. 2014. A statistical framework for inferring the influence of conspecifics on movement behaviour. Methods in Ecology and Evolution 5:183-189.

Doligez, B., C. Cadet, E. Danchin, and T. Boulinier. 2003. When to use public information for breeding habitat selection? The role of environmental predictability and density dependence. Animal Behaviour 66:973-988.

Doligez, B., E. Danchin, and J. Clobert. 2002. Public information and breeding habitat selection in a wild bird population. Science 297:1168-1170.

Doligez, B., E. Danchin, J. Clobert, and L. Gustafsson. 1999. The use of conspecific reproductive success for breeding habitat selection in a non-colonial, hole-nesting species, the collared flycatcher. Journal of Animal Ecology 68:1193-1206.

Doligez, B., T. Pärt, and E. Danchin. 2004a. Prospecting in the collared flycatcher: gathering public information for future breeding habitat selection? Animal Behaviour 67:457-466.

Doligez, B., T. Pärt, E. Danchin, J. Clobert, and L. Gustafsson. 2004b. Availability and use of public information and conspecific density for settlement decisions in the collared flycatcher. Journal of Animal Ecology 73:75-87.

Ens, B. J., M. Kersten, A. Brenninkmeijer, and J. B. Hulscher. 1992. Territory quality, parental effort and reproductive success of oystercatchers (Haematopus ostralegus). Journal of Animal Ecology 61:703-715.

Farrell, S. L., M. L. Morrison, A. J. Campomizzi, and R. N. Wilkins. 2012. Conspecific cues and breeding habitat selection in an endangered woodland warbler. Journal of Animal Ecology 81:1056-1064.

Fletcher, R. J., Jr. 2006. Emergent properties of conspecific attraction in fragmented landscapes. American Naturalist 168:207-219.
Fletcher, R. J., Jr. 2007. Species interactions and population density mediate the use of social cues for habitat selection. Journal of Animal Ecology 76:598-606.

Forsman, J. T., M. B. Hjernquist, and L. Gustafsson. 2009. Experimental evidence for the use of density based interspecific social information in forest birds. Ecography 32:539545.

Forsman, J. T., M. B. Hjernquist, J. Taipale, and L. Gustafsson. 2008. Competitor density cues for habitat quality facilitating habitat selection and investment decisions. Behavioral Ecology 19:539-545.

Forsman, J. T., and J.-T. Seppänen. 2011. Learning what (not) to do: testing rejection and copying of simulated heterospecific behavioural traits. Animal Behaviour 81:879-883.

Forsman, J. T., J.-T. Seppänen, and M. Mönkkönen. 2002. Positive fitness consequences of interspecific interaction with a potential competitor. Proceedings of the Royal Society B 269:1619-1623.

Forsman, J. T., and R. L. Thomson. 2008. Evidence of information collection from heterospecifics in cavity-nesting birds. Ibis 150:409-412.

Forsman, J. T., R. L. Thomson, and J.-T. Seppänen. 2007. Mechanisms and fitness effects of interspecific information use between migrant and resident birds. Behavioral Ecology 18:888-894.

Fretwell, S. D., and H. L. Lucas, Jr. 1969. On territorial behavior and other factors influencing habitat distribution in birds. I. Theoretical development. Acta Biotheoretica 19:1636.

Gustafsson, L. 1987. Interspecific competition lowers fitness in Collared Flycatchers Ficedula albicollis: an experimental demonstration. Ecology 68:291-296.

Hadfield, J. 2010. MCMC methods for Multi-response Generalised Linear Mixed Models: the MCMCglmm R Package. Journal of Statistical Software 33:1-22.

Hodge, M. A., and A. Storfer-Isser. 1997. Conspecific and heterospecific attraction: a mechanism of web-site selection leading to aggregation formation by web-building spiders. Ethology 103:815-826.

Hoi, H., A. Krištín, F. Valera, and C. Hoi. 2012. Traditional versus non-traditional nest-site choice: alternative decision strategies for nest-site selection. Oecologia 169:117-124.

Loukola, O. J., J. T. Seppänen, I. Krams, S. Torvinen, and J. T. Forsman. 2013. Observed fitness may affect niche overlap in competing species via selective social information use. American Naturalist 182:474-483.

MacArthur, R. H. 1972. Geographical ecology. patterns in the distribution of species. Harper and Row, New York, New York, USA.

Martin, P. R., and T. E. Martin. 2001. Ecological and fitness consequences of species coexistence: a removal experiment with wood warblers. Ecology 82:189-206.

Martin, T. E. 1996. Fitness costs of resource overlap among coexisting bird species. Nature 380:338-340.

Merilä, J., and D. A. Wiggins. 1995. Interspecific competition for nest holes causes adult mortality in the collared flycatcher. Condor 97:445-450.

Mönkkönen, M., R. Härdling, J. T. Forsman, and J. Tuomi. 1999. Evolution of heterospecific attraction: using other species as cues in habitat selection. Evolutionary Ecology 13: 91-104.

Mönkkönen, M., P. Helle, and K. Soppela. 1990. Numerical and behavioural responses of migrant passerines to experimental manipulation of resident tits (Parus spp.): heterospecific attraction in northern breeding bird communities? Oecologia 85:218-225.

Muller, K. L., J. A. Stamps, V. V. Krishnan, and N. H. Willits. 1997. The effects of conspecific attraction and habitat quality on habitat selection in territorial birds (Troglodytes aedon). American Naturalist 150:650-661. 
Nocera, J. J., G. J. Forbes, and L.-A. Giraldeau. 2006. Inadvertent social information in breeding site selection of natal dispersing birds. Proceedings of the Royal Society B 273:349-355.

Parejo, D., E. Danchin, and J. M. Avilés. 2005. The heterospecific habitat copying hypothesis: can competitors indicate habitat quality? Behavioral Ecology 16:96-105.

Parejo, D., E. Danchin, N. Silva, J. F. White, A. N. Dreiss, and J. M. Avilés. 2008. Do great tits rely on inadvertent social information from blue tits? A habitat selection experiment. Behavioral Ecology and Sociobiology 62:1569-1579.

Parejo, D., J. White, J. Clobert, A. Dreiss, and E. Danchin. 2007. Blue tits use fledgling quantity and quality as public information in breeding site choice. Ecology 88:2373-2382.

Pärt, T. 2001. The effects of territory quality on age-dependent reproductive performance in the northern wheatear, Oenanthe oenanthe. Animal Behaviour 62:379-388.

Pärt, T., D. Arlt, B. Doligez, M. Low, and A. Qvarnström. 2011. Prospectors combine social and environmental information to improve habitat selection and breeding success in the subsequent year. Journal of Animal Ecology 80:12271235.

Pärt, T., and B. Doligez. 2003. Gathering public information for habitat selection: prospecting birds cue on parental activity. Proceedings of the Royal Society B 270:1809-1813.

Pärt, T., and L. Gustafsson. 1989. Breeding dispersal in the Collared Flycatcher (Ficedula albicollis): possible causes and reproductive consequences. Journal of Animal Ecology 58: 305-320.

Plummer, M., N. Best, K. Cowles, and K. Vines. 2006. CODA: convergence diagnosis and output analysis for MCMC. R News 6:7-11.

R Development Core Team. 2011. R: A language and environment for statistical computing. R Foundation for Statistical Computing, Vienna, Austria.

Sasvári, L., J. Török, and L. Tóth. 1987. Density dependent effects between three competitive bird species. Oecologia 72: $127-130$.
Sebastián-Gonzáles, E., J. A. Sánchez-Zapata, F. Botella, and O. Ovaskainen. 2010. Testing the heterospecific attraction hypothesis with time-series data on species co-occurrence. Proceedings of the Royal Society B 277:2983-2990.

Seppänen, J.-T., and J. T. Forsman. 2007. Interspecific social learning: novel preference can be acquired from a competing species. Current Biology 17:1-5.

Seppänen, J.-T., J. T. Forsman, M. Mönkkönen, I. Krams, and T. Salmi. 2011. New behavioural trait adopted or rejected by observing heterospecific tutor fitness. Proceedings of the Royal Society B 278:1736-1741.

Seppänen, J.-T., J. T. Forsman, M. Mönkkönen, and R. L. Thomson. 2007. Social information use is a process across time, space and ecology, reaching heterospecifics. Ecology 88: $1622-1633$

Siikamäki, P. 1998. Limitation of reproductive success by food availability and breeding time in pied flycatchers. Ecology 79: 1789-1796.

Stamps, J. 1988. Conspecific attraction and aggregation in territorial species. American Naturalist 131:329-347.

Stöhr, S. 1998. Evolution of mate-choice copying: a dynamic model. Animal Behaviour 55:893-903.

Stokes, D. L., and P. D. Boersma. 1998. Nest-site characteristics and reproductive success in Magellanic penguins (Spheniscus magellanicus). The Auk 115:34-49.

Thomson, R. L., J. T. Forsman, and M. Mönkkönen. 2003. Positive interactions between migrant and resident birds: testing the heterospecific attraction hypothesis. Oecologia 134:431-438.

Valone, T. J., and J. J. Templeton. 2002. Public information for the assessment of quality: a widespread social phenomenon. Philosophical Transactions of the Royal Society B 357:15491557.

Wagner, R. H., and E. Danchin. 2003. Conspecific copying: a general mechanism of social aggregation. Animal Behaviour 65:405-408.

Wiggins, D. A., T. Pärt, and L. Gustafsson. 1994. Seasonal decline in collared flycatcher Ficedula albicollis reproductive success: an experimental approach. Oikos 70:359-364.

\section{Supplemental Material}

\section{Ecological Archives}

Appendices A and B are available online: http://dx.doi.org/10.1890/13-2103.1.sm 